\title{
Optimality conditions using convexifactors for a multiobjective fractional bilevel programming problem
}

\author{
Bhawna Kohli
}

\begin{abstract}
In this paper, a multiobjective fractional bilevel programming problem is considered and optimality conditions using the concept of convexifactors are established for it. For this purpose, a suitable constraint qualification in terms of convexifactors is introduced for the problem. Further in the paper, notions of asymptotic pseudoconvexity and asymptotic quasiconvexity in terms of convexifactors are given and using them sufficient optimality conditions are derived.
\end{abstract}

Keywords. Multiobjective fractional bilevel programming problem, convexifactors, value function, constraint qualifications

\section{Introduction}

Bilevel programming problem (BLPP) consists of two optimization problems where variables of the first (or upper-level or leader's) problem are the parameters of the second (or lower-level or follower's) problem and the optimal solution of the second is needed to calculate the objective function value of the first problem. They play an important role not only theoretically but also practically. During past many years, researchers like Bard [1, 2], Dempe [12, 13], Outrata [31], Suneja and Kohli [32] and many others have worked for the development of optimality conditions for this problem. For applications and recent developments on bilevel programming problems one can see Bard [3], Dempe [14].

Multiobjective bilevel programming problem, where one of the objective functions of the upper-level problem and/or the lower-level problem is a vector function, needs to be investigated intensively as it is demanded from the point-of-view of applications. They have been studied in the literature by authors such as Bouibed et al. [8], Ye [36], Bonnel [5], Bonnel and Morgan [6, 7], Tung [35], Luu and Mai [29] and others. Recently, Luu and Mai [29] developed necessary and sufficient efficiency conditions for multiobjective bilevel programming problem via convexifactors.

Fractional programming problems are of great importance in transportation, production, information theory and numerical analysis. Fractional bilevel programming problems have been studied in the literature by Calvete and Gale [9, 10, 11]. Very recently, Luu and Linh [30] obtained optimality and duality results for multiobjective fractional problems using convexifactors. In this paper, we have combined these two important problems, multiobjective fractional programming

Received date: August 15, 2020; Published online: November 10, 2021.

2010 Mathematics Subject Classification. 90C29, 90C32, 90C46.

Corresponding author: Bhawna Kohli. 
problem and bilevel programming problem to develop optimality conditions for multiobjective fractional bilevel programming problem where the upper-level problem is a multiobjective fractional programming problem and the lower-level problem is a scalar optimization problem.

Bilevel programming problems do not satisfy the usual constraint qualifications (CQs) such as Slater CQ, Mangasarian-Fromovitz CQ etc. It has been a subject of great challenge towards researchers to find a suitable CQ so as to develop Karush-Kuhn-Tucker (KKT) type necessary optimality conditions. Work has been done in this direction. Ye [37] introduced CQs in terms of Michel-Penot subdifferential and used them to obtain optimality conditions for bilevel programming problem. Later, Ye [38] introduced nonsmooth constraint qualifications for bilevel programming problem and used them to derive KKT type necessary optimality conditions. Dempe et al. [15] obtained KKT type optimality conditions for optimistic bilevel programming problem under the assumption of partial calmness CQ. Kohli [25] introduced two versions of Abadie CQ in terms of convexifactors and Clarke subdifferential and employed the weaker one to develop new necessary KKT type optimality conditions for optimistic bilevel programming problem using an upper estimate of Clarke subdifferential of value function and the concept of convexifactors.

In this paper, our aim is to prove KKT type optimality conditions for multiobjective fractional bilevel programming problem (MOFBLPP). For that, we have introduced a nonsmooth CQ namely $\partial^{*}$-MOFBLPP CQ in terms of convexifactors on the lines of Jourani [21]. Using weighted sum scalarization method, first we transform multiobjective fractional bilevel programming problem to a scalar optimization problem and after that we derive necessary optimality conditions. Further in the paper, we define asymptotic pseudoconvex and asymptotic quasiconvex functions in terms of convexifactors. Finally, under the assumption of these functions, we derive sufficient optimality conditions for the problem. Optimality conditions developed in terms of generalized subdifferential, convexifactor are more general since in the form of a convexifactor we get a smaller set. These convexifactors are tools of nonsmooth analysis and were given by Demyanov [16]. They were further studied by Jeyakumar and Luc [20], Dutta and Chandra [17, 18], Li and Zhang [27], Luu [28], Suneja and Kohli [32, 33, 34], Kohli [26], Kabgani and Soleimani-damaneh [22, 23], Kabgani et al. [24] and others. Since for locally Lipschitz functions, many well known subdifferentials such as Clarke subdifferential, Michel-Penot subdifferential etc may often contain convex hull of a convexifactor (Example $2.1[17,20]$ ), hence using convexifactors, we obtain sharp optimality conditions than those using Clarke, Michel-Penot subdifferentials etc.

The paper is organized as follows. In Section 2, we give definitions of convexifactors along with some basic results which will be used in the proof of main results. MOFBLPP has been studied in Section 3. In Section 4, we define asymptotic pseudoconvex and asymptotic quasiconvex functions in terms of convexifactors and also we give a constraint qualification using the concept of convexifactors. Section 5 is devoted to the study of necessary optimality conditions for MOFBLPP. Finally, in Section 6, we give sufficient optimality conditions for the problem.

\section{Convexifactors}

We begin by defining upper and lower Dini derivatives as follows:

Definition 1. Let $F: \mathbb{R}^{n_{1}} \rightarrow \mathbb{R} \cup\{ \pm \infty\}$ be an extended real valued function and let $x \in \mathbb{R}^{n_{1}}$ where $F(x)$ is finite. Then the upper and lower Dini derivatives of $F$ at $x$ in the direction $v$ are defined respectively by

$$
(F)_{d}^{+}(x, v):=\limsup _{t \rightarrow 0^{+}} \frac{F(x+t v)-F(x)}{t} \quad \text { and } \quad(F)_{d}^{-}(x, v):=\liminf _{t \rightarrow 0^{+}} \frac{F(x+t v)-F(x)}{t} .
$$


Dini derivatives may be finite as well as infinite. In particular, if $F$ is locally Lipschitz, both the upper and lower Dini derivatives are finite. For any set $A \subset \mathbb{R}^{n_{1}}$, the closure, convex hull and the closed convex hull of $A$ are denoted respectively by $\operatorname{cl} A$, conv $A$ and clconv $A$. We now give the definitions of convexifactors as given by Dutta and Chandra [17].

Definition 2. Let $F: \mathbb{R}^{n_{1}} \rightarrow \mathbb{R} \cup\{ \pm \infty\}$ be an extended real valued function and let $x \in \mathbb{R}^{n_{1}}$ where $F(x)$ is finite.

(i) $F$ is said to admit an upper convexifactor (UCF) $\partial^{u} F(x)$ at $x$ iff $\partial^{u} F(x) \subseteq \mathbb{R}^{n_{1}}$ is a closed set and

$$
(F)_{d}^{-}(x, v) \leq \sup _{x^{*} \in \partial^{u} F(x)}\left\langle x^{*}, v\right\rangle, \quad \text { for all } v \in \mathbb{R}^{n_{1}} .
$$

(ii) $F$ is said to admit a lower convexifactor (LCF) $\partial_{l} F(x)$ at $x$ iff $\partial_{l} F(x) \subseteq \mathbb{R}^{n_{1}}$ is a closed set and

$$
(F)_{d}^{+}(x, v) \geq \inf _{x^{*} \in \partial_{l} F(x)}\left\langle x^{*}, v\right\rangle, \quad \text { for all } v \in \mathbb{R}^{n_{1}} .
$$

(iii) $F$ is said to admit a convexifactor $(\mathrm{CF}) \partial^{*} F(x)$ at $x$ iff $\partial^{*} F(x)$ is both an (UCF) and (LCF) of $F$ at $x$.

(iv) $F$ is said to admit an upper semiregular convexifactor (USRCF) $\partial^{u s} F(x)$ at $x$ iff $\partial^{u s} F(x) \subseteq$ $\mathbb{R}^{n_{1}}$ is a closed set and

$$
(F)_{d}^{+}(x, v) \leq \sup _{x^{*} \in \partial^{u s} F(x)}\left\langle x^{*}, v\right\rangle, \quad \text { for all } v \in \mathbb{R}^{n_{1}} .
$$

In particular, if equality holds in above, then, $\partial^{u s} F(x)$ is called an upper regular convexifactor (URCF) of $F$ at $x$.

(v) $F$ is said to admit a lower semiregular convexifactor (LSRCF) $\partial_{l s} F(x)$ at $x$ iff $\partial_{l s} F(x) \subseteq \mathbb{R}^{n_{1}}$ is a closed set and

$$
(F)_{d}^{-}(x, v) \geq \inf _{x^{*} \in \partial_{l s} F(x)}\left\langle x^{*}, v\right\rangle, \quad \text { for all } v \in \mathbb{R}^{n_{1}} .
$$

In particular, if equality holds in above, then, $\partial_{l s} F(x)$ is called a lower regular convexifactor (LRCF) of $F$ at $x$.

It may be noted that convexifactors are always closed sets but not necessarily convex or compact (Jeyakumar and Luc [20], Dutta and Chandra [17, 18]) though the most well known subdifferentials such as Clarke, Michel-Penot etc are always convex and compact. Because of these relaxations, convexifactors can be easily applied to a large class of nonsmooth problems.

We now give one of the calculus rules given by Jeyakumar and Luc [20] for convexifactors in the form of the following remark.

Remark 1. Assume that the functions $f, g: \mathbb{R}^{n_{1}} \rightarrow \mathbb{R}$ admit UCFs $\partial^{u} f(x)$ and $\partial^{u} g(x)$ at $x$, respectively, and that one of the CFs is upper regular at $x$, then $\partial^{u} f(x)+\partial^{u} g(x)$ is an UCF of $f+g$ at $x$.

Similarly, if the functions $f, g: \mathbb{R}^{n_{1}} \rightarrow \mathbb{R}$ admit LCFs $\partial_{l} f(x)$ and $\partial_{l} g(x)$ at $x$, respectively, and that one of the CFs is lower regular at $x$, then $\partial_{l} f(x)+\partial_{l} g(x)$ is a LCF of $f+g$ at $x$.

The following lemma is crucial for our investigations. 
Lemma 2.1 (Lemma 2.1 (b) and (c), Li and Zhang [27]). Let $\partial^{u} f(x), \partial_{l} f(x)$ and $\partial^{*} f(x)$ respectively be UCF, LCF and CF of $f$ at $x$. Then,

(i) $\forall \lambda<0, \lambda \partial^{u} f(x)$ and $\lambda \partial_{l} f(x)$ respectively are $L C F$ and $U C F$ of $\lambda f$ at $x$.

(ii) $\forall \lambda \in \mathbb{R}, \lambda \partial^{*} f(x)$ is a $C F$ of $\lambda f$ at $x$.

Lemma 2.1(i) is valid for semiregular and regular CFs also.

Remark 2. Let $\partial^{u s} f(x)$ and $\partial_{l s} f(x)$ respectively be USRCF and LSRCF of $f$ at $x$. Then, $\forall \lambda<0, \lambda \partial^{u s} f(x)$ and $\lambda \partial_{l s} f(x)$ respectively are LSRCF and USRCF of $\lambda f$ at $x$.

Same holds for URCF and LRCF of $\lambda f$ at $x$ also.

Proof. Using definitions of upper (lower) dini derivatives and of limit superior (limit inferior), we can see that $\forall \lambda<0$,

(i) $(\lambda f)_{d}^{-}(x, v)=\lambda(f)_{d}^{+}(x, v)$, and

(ii) $(\lambda f)_{d}^{+}(x, v)=\lambda(f)_{d}^{-}(x, v)$, for all $v \in \mathbb{R}^{n_{1}}$.

Since $\partial^{u s} f(x)$ is an USRCF of $f$ at $x$, we have

$$
\begin{aligned}
& (f)_{d}^{+}(x, v) \leq \sup _{x^{*} \in \partial^{u s} f(x)}\left\langle x^{*}, v\right\rangle, \quad \text { for all } v \in \mathbb{R}^{n_{1}} \\
\Rightarrow \quad & \lambda(f)_{d}^{+}(x, v) \geq \lambda \sup _{x^{*} \in \partial^{u s} f(x)}\left\langle x^{*}, v\right\rangle, \quad \text { as } \lambda<0
\end{aligned}
$$

Using (i), we get

$$
\begin{aligned}
(\lambda f)_{d}^{-}(x, v) & \geq-\mu \sup _{x^{*} \in \partial^{u s} f(x)}\left\langle x^{*}, v\right\rangle, \quad \text { as } \lambda=-\mu, \mu>0 \\
& =-\sup _{x^{*} \in \partial^{u s} f(x)}\left\langle\mu x^{*}, v\right\rangle \\
& =\inf _{x^{*} \in \partial^{u s} f(x)}-\left\langle\mu x^{*}, v\right\rangle \\
& =\inf _{\xi \in \lambda \partial^{u s} f(x)}\langle\xi, v\rangle .
\end{aligned}
$$

That is,

$$
(\lambda f)_{d}^{-}(x, v) \geq \inf _{\xi \in \lambda \partial^{u s} f(x)}\langle\xi, v\rangle, \quad \text { for all } v \in \mathbb{R}^{n_{1}} .
$$

Thus $\lambda \partial^{u s} f(x)$ is a LSRCF of $\lambda f$ at $x$.

Similarly, using (ii) we can prove that $\lambda \partial_{l s} f(x)$ is an USRCF of $\lambda f$ at $x$.

Lemma 2.2 (Proposition 4.1, Jeyakumar and Luc [20]). Suppose that the function $f: \mathbb{R}^{n_{1}} \rightarrow \mathbb{R}$ admits a $C F \partial^{*} f(x)$ at $x \in \mathbb{R}^{n_{1}}$. If $f$ attains its extremum at $x$, then

$$
0 \in \operatorname{clconv}\left(\partial^{*} f(x)\right)
$$




\section{Multiobjective fractional bilevel programming problem}

In this section, we study the multiobjective fractional bilevel programming problem (MOFBLPP) which is defined as follows:

$$
\begin{array}{ll}
(\operatorname{MOFBLPP}) \quad & \underset{x, y}{\operatorname{Minimize}}\left(\frac{F_{1}(x, y)}{F_{1}^{\prime}(x, y)}, \ldots, \frac{F_{p}(x, y)}{F_{p}^{\prime}(x, y)}\right) \\
& \text { subject to } G_{j}(x, y) \leq 0, j \in J, y \in \psi(x),
\end{array}
$$

where, for each $x \in \mathbb{R}^{n_{1}}, \psi(x)$ is the set of the optimal solutions to the following optimization problem

$$
\begin{aligned}
& \underset{y}{\operatorname{Minimize}} f(x, y) \\
& \text { subject to } g_{i}(x, y) \leq 0, i \in I,
\end{aligned}
$$

where $F_{k}, F_{k}^{\prime}: \mathbb{R}^{n_{1}} \times \mathbb{R}^{n_{2}} \rightarrow \mathbb{R}, k=1, \ldots, p, f: \mathbb{R}^{n_{1}} \times \mathbb{R}^{n_{2}} \rightarrow \mathbb{R}, G_{j}: \mathbb{R}^{n_{1}} \times \mathbb{R}^{n_{2}} \rightarrow \mathbb{R}$, $j \in J:=\left\{1,2, \ldots, m_{2}\right\}$ and $g_{i}: \mathbb{R}^{n_{1}} \times \mathbb{R}^{n_{2}} \rightarrow \mathbb{R}, i \in I:=\left\{1,2, \ldots, m_{1}\right\} ; n_{i}$ and $m_{i}, i:=1,2$ are integers with $n_{i} \geq 1$ and $m_{i} \geq 1 . F_{k}(x, y) \geq 0, F_{k}^{\prime}(x, y)>0$ for all $k=1, \ldots, p$ and $(x, y) \in \mathbb{R}^{n_{1}} \times \mathbb{R}^{n_{2}}, f$ and $g_{i}, i \in I$ are continuous, convex and

$$
\psi(x):=\underset{y}{\operatorname{argmin}}\left\{f(x, y): g_{i}(x, y) \leq 0, i \in I\right\} .
$$

So, the idea is that, first, the leader fixes his choice $x$, and after that, the follower minimizes his objective function based on this choice and returns the solution $y=y(x)$ to the leader, who then uses it to minimize his objective function. If the lower-level problem has a unique optimal solution for all $x \in \mathbb{R}^{n_{1}}$, MOFBLPP is well defined, but if it has multiple solutions for a given $x$, then the upper-level objective becomes a set-valued map. To overcome this difficulty, two solution concepts namely the optimistic solution and the pessimistic solution have been discussed in the literature. In this paper, we follow optimistic approach. According to this approach, the leader presupposes cooperation of the follower in the sense that the latter will choose, each time that solution in $\psi(x)$ which is best suited with respect to the leader's objective function.

To convert BLPP into a single level programming problem, commonly three approaches have been discussed in the literature. Here, we adopt the optimal value reformulation initiated by Outrata [31] according to which multiobjective fractional bilevel programming problem can be converted into a single level mathematical programming problem with the help of the value function of the lower-level problem given by

$$
V(x):=\min _{y}\left\{f(x, y): g_{i}(x, y) \leq 0, i \in I, y \in \mathbb{R}^{n_{2}}\right\} .
$$

Then the reformulated multiobjective fractional bilevel programming problem (RMOFBLPP) is given as:

$$
\begin{gathered}
(\mathrm{RMOFBLPP}) \quad \underset{x, y}{\operatorname{Minimize}}\left(\frac{F_{1}(x, y)}{F_{1}^{\prime}(x, y)}, \ldots, \frac{F_{p}(x, y)}{F_{p}^{\prime}(x, y)}\right) \\
\text { subject to } f(x, y)-V(x) \leq 0 \\
g_{i}(x, y) \leq 0, i \in I, \\
G_{j}(x, y) \leq 0, j \in J, \\
(x, y) \in \mathbb{R}^{n_{1}} \times \mathbb{R}^{n_{2}} .
\end{gathered}
$$


Let $\phi(x, y):=\left(\frac{F_{1}(x, y)}{F_{1}^{\prime}(x, y)}, \ldots, \frac{F_{p}(x, y)}{F_{p}^{\prime}(x, y)}\right)$.

Let $E \subseteq \mathbb{R}^{n_{1}} \times \mathbb{R}^{n_{2}}$ denote the feasible set for RMOFBLPP, that is,

$$
E:=\left\{(x, y) \in \mathbb{R}^{n_{1}} \times \mathbb{R}^{n_{2}} \mid f(x, y)-V(x) \leq 0, g_{i}(x, y) \leq 0, i \in I, G_{j}(x, y) \leq 0, j \in J\right\} .
$$

Remark 3. On the lines of the remark made by Gadhi and Dempe [19], we can say that the problems MOFBLPP and RMOFBLPP are fully equivalent both with respect to local and global weak efficient solutions.

Now we define the notions of weak efficient and local weak efficient solutions of MOFBLPP.

Definition 3. $(\bar{x}, \bar{y}) \in E$ is a weak efficient solution of MOFBLPP if there does not exist any feasible solution $(x, y) \in E$ such that

$$
\frac{F_{k}(x, y)}{F_{k}^{\prime}(x, y)}<\frac{F_{k}(\bar{x}, \bar{y})}{F_{k}^{\prime}(\bar{x}, \bar{y})}, \quad k=1,2, \ldots, p .
$$

Definition 4. $(\bar{x}, \bar{y}) \in E$ is a local weak efficient solution of MOFBLPP if there exist neighbourhoods $U_{0}$ of $\bar{x}$ and $V_{0}$ of $\bar{y}$ such that for any feasible solution $(x, y) \in\left(U_{0} \times V_{0}\right) \cap E$, the following does not hold

$$
\frac{F_{k}(x, y)}{F_{k}^{\prime}(x, y)}<\frac{F_{k}(\bar{x}, \bar{y})}{F_{k}^{\prime}(\bar{x}, \bar{y})}, \quad k=1,2, \ldots, p .
$$

\section{$4 \quad \partial^{*}$-Asymptotic generalized convexity}

Let $F: \mathbb{R}^{n_{1}} \times \mathbb{R}^{n_{2}} \rightarrow \mathbb{R}$ be a function. Let $F$ admits a $(\mathrm{CF}) \partial^{*} F(\bar{x}, \bar{y})$ at $(\bar{x}, \bar{y})$.

We now define the notions of $\partial^{*}$-asymptotic generalized convex functions in terms of convexifactors on the lines of Luu [28]:

Definition 5. The function $F$ is said to be $\partial^{*}$-asymptotic pseudoconvex at $(\bar{x}, \bar{y})$ iff for all $(x, y) \in \mathbb{R}^{n_{1}} \times \mathbb{R}^{n_{2}}$

$$
F(x, y)<F(\bar{x}, \bar{y}) \Rightarrow\left\langle\xi^{*},(x-\bar{x}, y-\bar{y})\right\rangle<0 \quad \text { for all } \xi^{*} \in \operatorname{clconv} \partial^{*} F(\bar{x}, \bar{y}) .
$$

Remark 4. (i) If $F$ is a differentiable function and $\partial^{*} F(\bar{x}, \bar{y})$ is an upper regular convexifactor of $F$ at $(\bar{x}, \bar{y})$, then, $\partial^{*} F(\bar{x}, \bar{y})=\{\nabla F(\bar{x}, \bar{y})\}$ and the above definition reduces to the definition of pseudoconvex function.

(ii) If $F$ is a locally Lipschitz function and clconv $\partial^{*} F(\bar{x}, \bar{y})=\partial^{c} F(\bar{x}, \bar{y})$, where $\partial^{c} F(\bar{x}, \bar{y})$ is the Clarke subdifferential, then, the above definition reduces to the definition of $\partial^{c}$ pseudoconvex function given by Bector et al. [4].

Definition 6. The function $F$ is said to be $\partial^{*}$-asymptotic quasiconvex at $(\bar{x}, \bar{y})$ iff for all $(x, y) \in$ $\mathbb{R}^{n_{1}} \times \mathbb{R}^{n_{2}}$

$$
F(x, y) \leq F(\bar{x}, \bar{y}) \Rightarrow\left\langle\xi^{*},(x-\bar{x}, y-\bar{y})\right\rangle \leq 0 \quad \text { for all } \xi^{*} \in \operatorname{clconv} \partial^{*} F(\bar{x}, \bar{y}) .
$$

Remark 5. (i) If $F$ is a differentiable function and $\partial^{*} F(\bar{x}, \bar{y})$ is an upper regular convexifactor of $F$ at $(\bar{x}, \bar{y})$, then, $\partial^{*} F(\bar{x}, \bar{y})=\{\nabla F(\bar{x}, \bar{y})\}$ and the above definition reduces to the definition of quasiconvex function. 
(ii) If $F$ is a locally Lipschitz function and clconv $\partial^{*} F(\bar{x}, \bar{y})=\partial^{c} F(\bar{x}, \bar{y})$, then, the above definition reduces to the definition of $\partial^{c}$-quasiconvex function given by Bector et al. [4].

Now we give an example of $\partial^{*}$-asymptotic quasiconvex function.

Example 1. Let $F: \mathbb{R} \times \mathbb{R} \rightarrow \mathbb{R}$ be a function defined by

$$
F(x, y)= \begin{cases}\sqrt{x^{2} y}, & x \geq 0, y>0, \\ x, & x \geq 0, y=0, \\ \sqrt{-x y}, & x \geq 0, y<0 \\ -\sqrt{-x y^{2}}, & x<0, y \leq 0 \\ \sqrt{x^{2} y}, & x<0, y>0 .\end{cases}
$$

Convexifactor of $F$ at $(0,0)$ is given by

$$
\partial^{*} F(0,0)=\left\{\left(x^{*}+\frac{1}{n}, \frac{1}{n}\right): x^{*} \geq 0, n \in N\right\} \cup\left\{\left(x^{*}, 0\right): x^{*} \geq 0\right\} .
$$

$F$ is $\partial^{*}$-asymptotic quasiconvex at $(\bar{x}, \bar{y})=(0,0)$.

Following is the example of $\partial^{*}$-asymptotic pseudoconvex function.

Example 2. Let $F: \mathbb{R} \times \mathbb{R} \rightarrow \mathbb{R}$ be a function defined by

$$
F(x, y)= \begin{cases}-\sqrt{-x}-\sqrt{-y}, & x \leq 0, y \leq 0 \\ 0, & x>0, y<0 \\ \sqrt{-x}, & x<0, y>0 \\ \sqrt{x}+\sqrt{y}, & x \geq 0, y \geq 0 \sim\{x=0, y=0\}\end{cases}
$$

Convexifactor of $F$ at $(0,0)$ is given by

$$
\partial^{*} F(0,0)=\left\{\left(x^{*}+\frac{1}{n}, y^{*}+\frac{1}{n}\right): x^{*}>0, y^{*}>0, n \in N\right\} .
$$

$F$ is $\partial^{*}$-asymptotic pseudoconvex at $(\bar{x}, \bar{y})=(0,0)$ as

$$
\begin{aligned}
F(x, y)<F(0,0) \Rightarrow & \text { (i) } x<0, y<0 \\
& \text { or } \\
& \text { (ii) } x<0, y=0 \\
& \text { or } \\
& \text { (iii) } x=0, y<0
\end{aligned}
$$

To show

$$
\langle\xi,(x-0, y-0)\rangle<0, \text { for all } \xi \in \operatorname{clconv} \partial^{*} F(0,0)
$$

where

$$
\begin{aligned}
& \xi=\lim _{n \rightarrow \infty} \xi_{n}, \quad \text { for some } \xi_{n} \in \operatorname{conv} \partial^{*} F(0,0) \\
& \xi_{n}=\left(\xi_{1 n}, \xi_{2 n}\right)=\lambda\left(x^{*}+\frac{1}{n}, y^{*}+\frac{1}{n}\right)+(1-\lambda)\left(x_{1}^{*}+\frac{1}{n}, y_{1}^{*}+\frac{1}{n}\right), \quad 0 \leq \lambda \leq 1
\end{aligned}
$$


Taking limit, we get

$$
\xi=\lim _{n \rightarrow \infty} \xi_{n}=\left(\lambda x^{*}+(1-\lambda) x_{1}^{*}, \lambda y^{*}+(1-\lambda) y_{1}^{*}\right)
$$

Now

$$
\begin{aligned}
\langle\xi,(x, y)\rangle & =\left(\lambda x^{*}+(1-\lambda) x_{1}^{*}, \lambda y^{*}+(1-\lambda) y_{1}^{*}\right)\left(\begin{array}{l}
x \\
y
\end{array}\right) \\
& =\lambda x^{*} x+(1-\lambda) x_{1}^{*} x+\lambda y^{*} y+(1-\lambda) y_{1}^{*} y \\
& <0 \quad \text { for all cases (i), (ii) and (iii) }
\end{aligned}
$$

Hence

$$
F(x, y)<F(0,0) \Rightarrow\langle\xi,(x, y)\rangle<0 \quad \text { for all } \xi \in \operatorname{clconv} \partial^{*} F(0,0)
$$

We now introduce a nonsmooth CQ on the lines of CQ3 Jourani [21] in terms of convexifactors for RMOFBLPP. Let us define the following sets.

$$
I(\bar{x}, \bar{y})=\left\{i \in I: g_{i}(\bar{x}, \bar{y})=0\right\}, \quad J(\bar{x}, \bar{y})=\left\{j \in J: G_{j}(\bar{x}, \bar{y})=0\right\} .
$$

Let $(\bar{x}, \bar{y}) \in E$ be feasible for RMOFBLPP. We assume that the functions $f, g_{i}, i \in I(\bar{x}, \bar{y}), G_{j}$, $j \in J(\bar{x}, \bar{y})$ admit convexifactors $\partial^{*} f(\bar{x}, \bar{y}), \partial^{*} g_{i}(\bar{x}, \bar{y}), i \in I(\bar{x}, \bar{y})$ and $\partial^{*} G_{j}(\bar{x}, \bar{y}), j \in J(\bar{x}, \bar{y})$ respectively at $(\bar{x}, \bar{y})$.

The problem RMOFBLPP is said to satisfy $\partial^{*}$-MOFBLPP CQ at $(\bar{x}, \bar{y})$ if for every $\gamma \geq 0$, $\tau_{j} \geq 0, j \in J(\bar{x}, \bar{y}), \mu_{i} \geq 0, i \in I(\bar{x}, \bar{y})$ (not all zero)

$$
(0,0) \notin \mathrm{cl}\left[\begin{array}{l}
\gamma \operatorname{conv}\left(\partial^{*} f(\bar{x}, \bar{y})-\partial^{*} V(\bar{x}) \times\{0\}\right)+\sum_{j \in J(\bar{x}, \bar{y})} \tau_{j} \operatorname{conv} \partial^{*} G_{j}(\bar{x}, \bar{y}) \\
+\sum_{i \in I(\bar{x}, \bar{y})} \mu_{i} \operatorname{conv} \partial^{*} g_{i}(\bar{x}, \bar{y})
\end{array}\right] .
$$

\section{$5 \quad$ Necessary optimality conditions}

In this section, we derive KKT type necessary optimality conditions for MOFBLPP in the form of the following theorem.

Here, we define $\phi_{k}(\bar{x}, \bar{y})=\frac{F_{k}(\bar{x}, \bar{y})}{F_{k}^{\prime}(\bar{x}, \bar{y})}, \varphi_{k}(x, y)=F_{k}(x, y)-\phi_{k}(\bar{x}, \bar{y}) F_{k}^{\prime}(x, y), k=1,2, \ldots, p$.

Theorem 5.1. Let $(\bar{x}, \bar{y})$ be a local weak efficient solution of MOFBLPP. Suppose that $F_{k}$, $k=1,2, \ldots, p$ admit bounded convexifactors $\partial^{*} F_{k}(\bar{x}, \bar{y}), k=1,2, \ldots, p$ at $(\bar{x}, \bar{y})$ which are upper regular and $F_{k}^{\prime}, k=1,2, \ldots, p$ admit bounded convexifactors $\partial^{*} F_{k}^{\prime}(\bar{x}, \bar{y}), k=1,2, \ldots, p$ at $(\bar{x}, \bar{y})$ which are upper regular if $\phi_{k}(\bar{x}, \bar{y})>0$ and lower regular if $\phi_{k}(\bar{x}, \bar{y})<0$. Let $f$ admits a bounded convexifactor $\partial^{*} f(\bar{x}, \bar{y})$ at $(\bar{x}, \bar{y})$ which is upper regular and $G_{j}, j \in J, g_{i}, i \in I$ admit bounded convexifactors $\partial^{*} G_{j}(\bar{x}, \bar{y}), j \in J$ and $\partial^{*} g_{i}(\bar{x}, \bar{y}), i \in I$ respectively at $(\bar{x}, \bar{y})$. Suppose that $\partial^{*} \varphi_{k}, k=1,2, \ldots, p, \partial^{*} f, \partial^{*} G_{j}, j \in J, \partial^{*} g_{i}, i \in I$ are upper semicontinuous at $(\bar{x}, \bar{y})$. Also, assume that the functions $F_{k}, F_{k}^{\prime}, k=1,2, \ldots, p, G_{j}, j \in J$ are continuous and convex. Let $\partial^{*}$-MOFBLPP CQ be satisfied at $(\bar{x}, \bar{y})$. Assume that for some $k, k=1,2, \ldots, p$, $\partial^{*} F_{k}(\bar{x}, \bar{y})-\phi_{k}(\bar{x}, \bar{y}) \partial^{*} F_{k}^{\prime}(\bar{x}, \bar{y})$ is an upper regular convexifactor of $F_{k}-\phi_{k} F_{k}^{\prime}$ at $(\bar{x}, \bar{y})$ and for some $k_{0} \neq k, \partial^{*} F_{k_{0}}(\bar{x}, \bar{y})-\phi_{k_{0}}(\bar{x}, \bar{y}) \partial^{*} F_{k_{0}}^{\prime}(\bar{x}, \bar{y})$ is a lower regular convexifactor of $F_{k_{0}}-\phi_{k_{0}} F_{k_{0}}^{\prime}$ at $(\bar{x}, \bar{y})$. Suppose that

$$
\partial^{*}\left(\sum_{k=1}^{p} w_{k}\left(F_{k}-\phi_{k} F_{k}^{\prime}\right)\right)(\bar{x}, \bar{y})=\sum_{k=1}^{p} \partial^{*}\left(w_{k}\left(F_{k}-\phi_{k} F_{k}^{\prime}\right)\right)(\bar{x}, \bar{y})
$$




$$
=\sum_{k=1}^{p} w_{k}\left(\partial^{*} F_{k}(\bar{x}, \bar{y})-\phi_{k}(\bar{x}, \bar{y}) \partial^{*} F_{k}^{\prime}(\bar{x}, \bar{y})\right)
$$

where $w_{k}>0, k=1,2, \ldots, p$ and $\sum_{k=1}^{p} w_{k}=1$. Also, assume that $\partial^{*}(f(\bar{x}, \bar{y})-V(\bar{x}))=\partial^{*} f(\bar{x}, \bar{y})-$ $\partial^{*} V(\bar{x}) \times\{0\}$. Then, there exist scalars $w_{k}^{\prime} \geq 0,($ not all zero $), k=1,2, \ldots, p, \gamma \geq 0, \tau_{j} \geq 0$, $j \in J, \mu_{i} \geq 0, i \in I$ with $\sum_{k=1}^{p} w_{k}^{\prime}+\gamma+\sum_{j \in J} \tau_{j}+\sum_{i \in I} \mu_{i}=1$ such that

$$
(0,0) \in \operatorname{cl}\left[\begin{array}{l}
\sum_{k=1}^{p} w_{k}^{\prime}\left\{\operatorname{conv} \partial^{*} F_{k}(\bar{x}, \bar{y})-\phi_{k}(\bar{x}, \bar{y}) \operatorname{conv} \partial^{*} F_{k}^{\prime}(\bar{x}, \bar{y})\right\} \\
+\gamma \operatorname{conv}\left(\partial^{*} f(\bar{x}, \bar{y})-\partial^{*} V(\bar{x}) \times\{0\}\right) \\
+\sum_{j \in J} \tau_{j} \operatorname{conv} \partial^{*} G_{j}(\bar{x}, \bar{y})+\sum_{i \in I} \mu_{i} \operatorname{conv} \partial^{*} g_{i}(\bar{x}, \bar{y})
\end{array}\right] .
$$

Proof. Since $(\bar{x}, \bar{y})$ is a local weak efficient solution of MOFBLPP, hence by Remark $3,(\bar{x}, \bar{y})$ is a local weak efficient solution of RMOFBLPP.

Our proof consists of the following steps.

At first we will prove that $(\bar{x}, \bar{y})$ is a local weak efficient solution of $\left(\mathrm{P}_{1}\right)$

$$
\begin{aligned}
& \left(\mathrm{P}_{1}\right) \quad \underset{x, y}{\operatorname{Minimize}}\left(F_{1}(x, y)-\phi_{1}(\bar{x}, \bar{y}) F_{1}^{\prime}(x, y), \ldots, F_{p}(x, y)-\phi_{p}(\bar{x}, \bar{y}) F_{p}^{\prime}(x, y)\right) \\
& \text { subject to }(x, y) \in E .
\end{aligned}
$$

On contrary suppose that there exists $\left(x_{1}, y_{1}\right) \in\left(U_{0} \times V_{0}\right) \bigcap E$ such that

$$
F_{k}\left(x_{1}, y_{1}\right)-\phi_{k}(\bar{x}, \bar{y}) F_{k}^{\prime}\left(x_{1}, y_{1}\right)<F_{k}(\bar{x}, \bar{y})-\phi_{k}(\bar{x}, \bar{y}) F_{k}^{\prime}(\bar{x}, \bar{y}), \quad k=1,2, \ldots, p .
$$

Now

$$
F_{k}(\bar{x}, \bar{y})-\phi_{k}(\bar{x}, \bar{y}) F_{k}^{\prime}(\bar{x}, \bar{y})=0, \quad k=1,2, \ldots, p .
$$

Therefore, we have

$$
F_{k}\left(x_{1}, y_{1}\right)-\phi_{k}(\bar{x}, \bar{y}) F_{k}^{\prime}\left(x_{1}, y_{1}\right)<0, \quad k=1,2, \ldots, p .
$$

That is,

$$
\frac{F_{k}\left(x_{1}, y_{1}\right)}{F_{k}^{\prime}\left(x_{1}, y_{1}\right)}<\frac{F_{k}(\bar{x}, \bar{y})}{F_{k}^{\prime}(\bar{x}, \bar{y})}, \quad k=1,2, \ldots, p
$$

which is contradiction to the fact that $(\bar{x}, \bar{y})$ is a local weak efficient solution of RMOFBLPP.

Hence $(\bar{x}, \bar{y})$ is a local weak efficient solution of $\left(\mathrm{P}_{1}\right)$.

Next, we prove that $(\bar{x}, \bar{y})$ is a local optimal solution of the scalar problem $\left(\mathrm{P}_{2}\right)$.

By weighted sum approach, we have

$$
\begin{array}{r}
\left(\mathrm{P}_{2}\right) \underset{x, y}{\operatorname{Minimize}}\left(\sum_{k=1}^{p} w_{k}\left(F_{k}(x, y)-\phi_{k}(\bar{x}, \bar{y}) F_{k}^{\prime}(x, y)\right)\right) \\
w_{k}>0, k=1,2, \ldots, p, \sum_{k=1}^{p} w_{k}=1
\end{array}
$$




$$
\text { subject to }(x, y) \in E \text {. }
$$

Since $(\bar{x}, \bar{y})$ is a local weak efficient solution of $\left(\mathrm{P}_{1}\right)$, there does not exist any feasible solution $(x, y) \in\left(U_{0} \times V_{0}\right) \cap E$ such that the following hold

$$
F_{k}(x, y)-\phi_{k}(\bar{x}, \bar{y}) F_{k}^{\prime}(x, y)<F_{k}(\bar{x}, \bar{y})-\phi_{k}(\bar{x}, \bar{y}) F_{k}^{\prime}(\bar{x}, \bar{y}), \quad k=1,2, \ldots, p,
$$

where $F_{k}^{\prime}(x, y)>0$, for all $k=1,2, \ldots, p$ and $(x, y) \in \mathbb{R}^{n_{1}} \times \mathbb{R}^{n_{2}}$,

$$
\Rightarrow w_{k}\left(F_{k}(x, y)-\phi_{k}(\bar{x}, \bar{y}) F_{k}^{\prime}(x, y)\right)<w_{k}\left(F_{k}(\bar{x}, \bar{y})-\phi_{k}(\bar{x}, \bar{y}) F_{k}^{\prime}(\bar{x}, \bar{y})\right),
$$

as $w_{k}>0, k=1,2, \ldots, p$.

Thus, for any feasible solution $(x, y) \in\left(U_{0} \times V_{0}\right) \cap E$, following does not hold

$$
\sum_{k=1}^{p} w_{k}\left(F_{k}(x, y)-\phi_{k}(\bar{x}, \bar{y}) F_{k}^{\prime}(x, y)\right)<\sum_{k=1}^{p} w_{k}\left(F_{k}(\bar{x}, \bar{y})-\phi_{k}(\bar{x}, \bar{y}) F_{k}^{\prime}(\bar{x}, \bar{y})\right) .
$$

Therefore $(\bar{x}, \bar{y})$ is a local optimal solution of $\left(\mathrm{P}_{2}\right)$.

Since $(\bar{x}, \bar{y})$ solves $\left(\mathrm{P}_{2}\right)$, it solves the following unconstrained problem

$$
\underset{x, y}{\operatorname{Minimize}} H(x, y)=\max \left\{\begin{array}{c}
\sum_{k=1}^{p} w_{k} \varphi_{k}(x, y)-\sum_{k=1}^{p} w_{k} \varphi_{k}(\bar{x}, \bar{y}), f(x, y)-V(x), \\
G_{1}(x, y), \ldots, G_{m_{2}}(x, y), \\
g_{1}(x, y), \ldots, g_{m_{1}}(x, y)
\end{array}\right\} .
$$

Now,

$$
\sum_{k=1}^{p} w_{k} \varphi_{k}(\bar{x}, \bar{y})=0
$$

By Lemma 2.2, we have

$$
(0,0) \in \operatorname{clconv} \partial^{*} H(\bar{x}, \bar{y})
$$

where using Theorem 3.2 [17],

$$
\partial^{*} H(\bar{x}, \bar{y})=\operatorname{conv}\left\{\begin{array}{l}
\partial^{*}\left(\sum_{k=1}^{p} w_{k} \varphi_{k}(\bar{x}, \bar{y})\right) \cup \partial^{*}(f(\bar{x}, \bar{y})-V(\bar{x})) \cup \bigcup_{j \in J(\bar{x}, \bar{y})} \partial^{*} G_{j}(\bar{x}, \bar{y}) \\
\cup \bigcup_{i \in I(\bar{x}, \bar{y})} \partial^{*} g_{i}(\bar{x}, \bar{y})
\end{array}\right\} .
$$

Thus,

$$
(0,0) \in \operatorname{clconv}\left(\operatorname{conv}\left\{\begin{array}{l}
\partial^{*}\left(\sum_{k=1}^{p} w_{k} \varphi_{k}(\bar{x}, \bar{y})\right) \cup \partial^{*}(f(\bar{x}, \bar{y})-V(\bar{x})) \cup \bigcup_{j \in J(\bar{x}, \bar{y})} \partial^{*} G_{j}(\bar{x}, \bar{y}) \\
\cup \bigcup_{i \in I(\bar{x}, \bar{y})} \partial^{*} g_{i}(\bar{x}, \bar{y})
\end{array}\right\}\right),
$$

which implies that there exists a sequence

$$
\left(x_{n}, y_{n}\right) \in \operatorname{conv}\left(\operatorname{conv}\left\{\begin{array}{l}
\partial^{*}\left(\sum_{k=1}^{p} w_{k} \varphi_{k}(\bar{x}, \bar{y})\right) \cup \partial^{*}(f(\bar{x}, \bar{y})-V(\bar{x})) \cup \bigcup_{j \in J(\bar{x}, \bar{y})} \partial^{*} G_{j}(\bar{x}, \bar{y}) \\
\cup \bigcup_{i \in I(\bar{x}, \bar{y})} \partial^{*} g_{i}(\bar{x}, \bar{y})
\end{array}\right\}\right),
$$

such that $\left(x_{n}, y_{n}\right) \rightarrow(0,0)$ as $n \rightarrow \infty$. 
Since convexifactors are in general nonconvex sets, and there exist sequences of scalars $\left\{\lambda^{\prime n}\right\}, \lambda^{\prime n} \geq 0,\left\{\gamma^{n}\right\}, \gamma^{n} \geq 0,\left\{\tau_{j}^{n}\right\}, \tau_{j}^{n} \geq 0, j \in J(\bar{x}, \bar{y}),\left\{\mu_{i}^{n}\right\}, \mu_{i}^{n} \geq 0, i \in I(\bar{x}, \bar{y}), n \in N$ with $\lim _{n \rightarrow \infty}\left[\lambda^{\prime n}+\gamma^{n}+\sum_{j \in J(\bar{x}, \bar{y})} \tau_{j}^{n}+\sum_{i \in I(\bar{x}, \bar{y})} \mu_{i}^{n}\right]=1$ such that

$$
\begin{aligned}
\left(x_{n}, y_{n}\right) \in & \lambda^{\prime n} \operatorname{conv} \partial^{*}\left(\sum_{k=1}^{p} w_{k} \varphi_{k}(\bar{x}, \bar{y})\right)+\gamma^{n}\left(\operatorname{conv} \partial^{*}(f(\bar{x}, \bar{y})-V(\bar{x}))\right) \\
& +\sum_{j \in J(\bar{x}, \bar{y})} \tau_{j}^{n} \operatorname{conv} \partial^{*} G_{j}(\bar{x}, \bar{y})+\sum_{i \in I(\bar{x}, \bar{y})} \mu_{i}^{n} \operatorname{conv} \partial^{*} g_{i}(\bar{x}, \bar{y}) .
\end{aligned}
$$

That is,

$$
\begin{aligned}
\left(x_{n}, y_{n}\right) \in & \lambda^{\prime n} \operatorname{conv}\left(\sum_{k=1}^{p} w_{k}\left(\partial^{*} F_{k}(\bar{x}, \bar{y})-\phi_{k}(\bar{x}, \bar{y}) \partial^{*} F_{k}^{\prime}(\bar{x}, \bar{y})\right)\right) \\
& +\gamma^{n}\left(\operatorname{conv}\left(\partial^{*} f(\bar{x}, \bar{y})-\partial^{*} V(\bar{x}) \times\{0\}\right)\right) \\
& +\sum_{j \in J(\bar{x}, \bar{y})} \tau_{j}^{n} \operatorname{conv} \partial^{*} G_{j}(\bar{x}, \bar{y})+\sum_{i \in I(\bar{x}, \bar{y})} \mu_{i}^{n} \operatorname{conv} \partial^{*} g_{i}(\bar{x}, \bar{y}) .
\end{aligned}
$$

Using convex hull property of subsets $A_{1}$ and $A_{2}$ of $\mathbb{R}^{n_{1}}, \operatorname{conv}\left(A_{1}+A_{2}\right)=\operatorname{conv}\left(A_{1}\right)+\operatorname{conv}\left(A_{2}\right)$ and of a subset $A$ of $\mathbb{R}^{n_{1}}, \operatorname{conv}(\lambda A)=\lambda \operatorname{conv}(A), \lambda \in \mathbb{R}$, we get

$$
\begin{aligned}
\left(x_{n}, y_{n}\right) \in & \sum_{k=1}^{p} w_{k}^{\prime n} \operatorname{conv}\left\{\partial^{*} F_{k}(\bar{x}, \bar{y})-\phi_{k}(\bar{x}, \bar{y}) \partial^{*} F_{k}^{\prime}(\bar{x}, \bar{y})\right\} \\
& +\gamma^{n}\left(\operatorname{conv}\left(\partial^{*} f(\bar{x}, \bar{y})-\partial^{*} V(\bar{x}) \times\{0\}\right)\right) \\
& +\sum_{j \in J(\bar{x}, \bar{y})} \tau_{j}^{n} \operatorname{conv} \partial^{*} G_{j}(\bar{x}, \bar{y})+\sum_{i \in I(\bar{x}, \bar{y})} \mu_{i}^{n} \operatorname{conv} \partial^{*} g_{i}(\bar{x}, \bar{y})
\end{aligned}
$$

where

$$
w_{k}^{\prime n}=\lambda^{\prime n} w_{k}, k=1,2, \ldots, p, \lim _{n \rightarrow \infty}\left[\sum_{k=1}^{p} w_{k}^{\prime n}+\gamma^{n}+\sum_{j \in J(\bar{x}, \bar{y})} \tau_{j}^{n}+\sum_{i \in I(\bar{x}, \bar{y})} \mu_{i}^{n}\right]=1 .
$$

Since $\left\{w_{k}^{\prime n}\right\},\left\{\gamma^{n}\right\},\left\{\tau_{j}^{n}\right\}, j \in J(\bar{x}, \bar{y}),\left\{\mu_{i}^{n}\right\}, i \in I(\bar{x}, \bar{y})$ are bounded sequences, we may assume that $w_{k}^{\prime} n \rightarrow w_{k}^{\prime}, \gamma^{n} \rightarrow \gamma, \tau_{j}^{n} \rightarrow \tau_{j}, \mu_{i}^{n} \rightarrow \mu_{i}$ as $n \rightarrow \infty$.

Thus, we have

$$
(0,0) \in \operatorname{cl}\left[\begin{array}{c}
\sum_{k=1}^{p} w_{k}^{\prime} \operatorname{conv}\left\{\partial^{*} F_{k}(\bar{x}, \bar{y})-\phi_{k}(\bar{x}, \bar{y}) \partial^{*} F_{k}^{\prime}(\bar{x}, \bar{y})\right\} \\
+\gamma\left(\operatorname{conv}\left(\partial^{*} f(\bar{x}, \bar{y})-\partial^{*} V(\bar{x}) \times\{0\}\right)\right) \\
+\sum_{j \in J(\bar{x}, \bar{y})} \tau_{j} \operatorname{conv} \partial^{*} G_{j}(\bar{x}, \bar{y})+\sum_{i \in I(\bar{x}, \bar{y})} \mu_{i} \operatorname{conv} \partial^{*} g_{i}(\bar{x}, \bar{y})
\end{array}\right],
$$

with $\sum_{k=1}^{p} w_{k}^{\prime}+\gamma+\sum_{j \in J(\bar{x}, \bar{y})} \tau_{j}+\sum_{i \in I(\bar{x}, \bar{y})} \mu_{i}=1$. 
Again, using the convex hull property of subsets $A_{1}$ and $A_{2}$ of $\mathbb{R}^{n_{1}}, \operatorname{conv}\left(A_{1}+A_{2}\right)=$ $\operatorname{conv}\left(A_{1}\right)+\operatorname{conv}\left(A_{2}\right)$ and of a subset $A$ of $\mathbb{R}^{n_{1}}, \operatorname{conv}(\lambda A)=\lambda \operatorname{conv}(A), \lambda \in \mathbb{R}$, we get

$$
(0,0) \in \operatorname{cl}\left[\begin{array}{c}
\sum_{k=1}^{p} w_{k}^{\prime}\left\{\operatorname{conv} \partial^{*} F_{k}(\bar{x}, \bar{y})-\phi_{k}(\bar{x}, \bar{y}) \operatorname{conv} \partial^{*} F_{k}^{\prime}(\bar{x}, \bar{y})\right\} \\
+\gamma\left(\operatorname{conv}\left(\partial^{*} f(\bar{x}, \bar{y})-\partial^{*} V(\bar{x}) \times\{0\}\right)\right) \\
+\sum_{j \in J(\bar{x}, \bar{y})} \tau_{j} \operatorname{conv} \partial^{*} G_{j}(\bar{x}, \bar{y})+\sum_{i \in I(\bar{x}, \bar{y})} \mu_{i} \operatorname{conv} \partial^{*} g_{i}(\bar{x}, \bar{y})
\end{array}\right],
$$

with $\sum_{k=1}^{p} w_{k}^{\prime}+\gamma+\sum_{j \in J(\bar{x}, \bar{y})} \tau_{j}+\sum_{i \in I(\bar{x}, \bar{y})} \mu_{i}=1$.

Thus, there exist

$$
\begin{aligned}
& \xi_{k}^{n} \in \operatorname{conv} \partial^{*} F_{k}(\bar{x}, \bar{y}), \quad \xi_{k}^{\prime n} \in \operatorname{conv} \partial^{*} F_{k}^{\prime}(\bar{x}, \bar{y}), \quad k=1,2, \ldots, p \\
& \zeta^{n} \in \operatorname{conv}\left(\partial^{*} f(\bar{x}, \bar{y})-\partial^{*} V(\bar{x}) \times\{0\}\right), \\
& \eta_{i}^{n} \in \operatorname{conv} \partial^{*} g_{i}(\bar{x}, \bar{y}), \quad i \in I(\bar{x}, \bar{y}), \quad \gamma_{j}^{n} \in \operatorname{conv} \partial^{*} G_{j}(\bar{x}, \bar{y}), \quad j \in J(\bar{x}, \bar{y})
\end{aligned}
$$

such that

$$
\lim _{n \rightarrow \infty}\left[\sum_{k=1}^{p} w_{k}^{\prime}\left(\xi_{k}^{n}-\phi_{k}(\bar{x}, \bar{y}) \xi_{k}^{\prime n}\right)+\gamma \zeta^{n}+\sum_{i \in I(\bar{x}, \bar{y})} \mu_{i} \eta_{i}^{n}+\sum_{j \in J(\bar{x}, \bar{y})} \tau_{j} \gamma_{j}^{n}\right]=(0,0),
$$

with

$$
\sum_{k=1}^{p} w_{k}^{\prime}+\gamma+\sum_{j \in J(\bar{x}, \bar{y})} \tau_{j}+\sum_{i \in I(\bar{x}, \bar{y})} \mu_{i}=1
$$

For any arbitrary $\left(v_{1}, v_{2}\right) \in \mathbb{R}^{n_{1}} \times \mathbb{R}^{n_{2}}$, we have

$$
\lim _{n \rightarrow \infty}\left[\begin{array}{rl}
\sum_{k=1}^{p} w_{k}^{\prime} & \left\langle\left(\xi_{k}^{n}-\phi_{k}(\bar{x}, \bar{y}) \xi_{k}^{\prime n}\right),\left(v_{1}, v_{2}\right)\right\rangle+\gamma\left\langle\zeta^{n},\left(v_{1}, v_{2}\right)\right\rangle \\
& +\sum_{i \in I(\bar{x}, \bar{y})} \mu_{i}\left\langle\eta_{i}^{n},\left(v_{1}, v_{2}\right)\right\rangle+\sum_{j \in J(\bar{x}, \bar{y})} \tau_{j}\left\langle\gamma_{j}^{n},\left(v_{1}, v_{2}\right)\right\rangle
\end{array}\right]=0,
$$

with $\sum_{k=1}^{p} w_{k}^{\prime}+\gamma+\sum_{j \in J(\bar{x}, \bar{y})} \tau_{j}+\sum_{i \in I(\bar{x}, \bar{y})} \mu_{i}=1$.

Suppose that $w^{\prime}=\left(w_{1}^{\prime}, \ldots, w_{p}^{\prime}\right)=0$.

Then, we have, from (5.1),

$$
\lim _{n \rightarrow \infty}\left[\gamma\left\langle\zeta^{n},\left(v_{1}, v_{2}\right)\right\rangle+\sum_{i \in I(\bar{x}, \bar{y})} \mu_{i}\left\langle\eta_{i}^{n},\left(v_{1}, v_{2}\right)\right\rangle+\sum_{j \in J(\bar{x}, \bar{y})} \tau_{j}\left\langle\gamma_{j}^{n},\left(v_{1}, v_{2}\right)\right\rangle\right]=0,
$$

with $\gamma+\sum_{j \in J(\bar{x}, \bar{y})} \tau_{j}+\sum_{i \in I(\bar{x}, \bar{y})} \mu_{i}=1$.

From $\partial^{*}$-MOFBLPP CQ, for every $\gamma \geq 0, \tau_{j} \geq 0, j \in J(\bar{x}, \bar{y}), \mu_{i} \geq 0, i \in I(\bar{x}, \bar{y})$ (not all zero), for all, in particular for $\zeta^{n} \in \operatorname{conv}\left(\partial^{*} f(\bar{x}, \bar{y})-\partial^{*} V(\bar{x}) \times\{0\}\right), \eta_{i}^{n} \in \operatorname{conv} \partial^{*} g_{i}(\bar{x}, \bar{y})$, $i \in I(\bar{x}, \bar{y}), \gamma_{j}^{n} \in \operatorname{conv} \partial^{*} G_{j}(\bar{x}, \bar{y}), j \in J(\bar{x}, \bar{y})$, we have

$$
\lim _{n \rightarrow \infty}\left[\gamma \zeta^{n}+\sum_{j \in J(\bar{x}, \bar{y})} \tau_{j} \gamma_{j}^{n}+\sum_{i \in I(\bar{x}, \bar{y})} \mu_{i} \eta_{i}^{n}\right] \neq(0,0) .
$$


Thus, there exists $\left(v_{1}, v_{2}\right) \in \mathbb{R}^{n_{1}} \times \mathbb{R}^{n_{2}}$ such that

$$
\lim _{n \rightarrow \infty}\left[\gamma\left\langle\zeta^{n},\left(v_{1}, v_{2}\right)\right\rangle+\sum_{i \in I(\bar{x}, \bar{y})} \mu_{i}\left\langle\eta_{i}^{n},\left(v_{1}, v_{2}\right)\right\rangle+\sum_{j \in J(\bar{x}, \bar{y})} \tau_{j}\left\langle\gamma_{j}^{n},\left(v_{1}, v_{2}\right)\right\rangle\right] \neq 0,
$$

which contradicts (5.2). Hence $w^{\prime} \neq 0$. Putting $\mu_{i}=0$ for $i \notin I(\bar{x}, \bar{y})$ and $\tau_{j}=0$ for $j \notin J(\bar{x}, \bar{y})$ we establish the result.

Remark 6. If $\partial^{*} F_{k}(\bar{x}, \bar{y})$ are convexifactors of $F_{k}, k=1,2, \ldots, p$ at $(\bar{x}, \bar{y})$ which are upper regular and $\partial^{*} F_{k}^{\prime}(\bar{x}, \bar{y})$ are convexifactors of $F_{k}^{\prime}, k=1,2, \ldots, p$ at $(\bar{x}, \bar{y})$ which are upper regular if $\phi_{k}(\bar{x}, \bar{y})>0$ and lower regular if $\phi_{k}(\bar{x}, \bar{y})<0, k=1, \ldots, p$, then, using Remark 1 and Remark 2 , we have that $\partial^{*} F_{k}(\bar{x}, \bar{y})-\phi_{k}(\bar{x}, \bar{y}) \partial^{*} F_{k}^{\prime}(\bar{x}, \bar{y}), k=1,2, \ldots, p$ is a convexifactor of $F_{k}-\phi_{k} F_{k}^{\prime}$, $k=1,2, \ldots, p$ at $(\bar{x}, \bar{y})$. Also, if we assume that for some $k, k=1,2, \ldots, p, \partial^{*} F_{k}(\bar{x}, \bar{y})-$ $\phi_{k}(\bar{x}, \bar{y}) \partial^{*} F_{k}^{\prime}(\bar{x}, \bar{y})$ is an upper regular convexifactor of $F_{k}-\phi_{k} F_{k}^{\prime}$ at $(\bar{x}, \bar{y})$ and for some $k_{0} \neq k$, $\partial^{*} F_{k_{0}}(\bar{x}, \bar{y})-\phi_{k_{0}}(\bar{x}, \bar{y}) \partial^{*} F_{k_{0}}^{\prime}(\bar{x}, \bar{y})$ is a lower regular convexifactor of $F_{k_{0}}-\phi_{k_{0}} F_{k_{0}}^{\prime}$ at $(\bar{x}, \bar{y})$, then, using Remark 1, Lemma 2.1(ii) and above mentioned fact, we have that

$$
\begin{aligned}
\partial^{*}\left(\sum_{k=1}^{p} w_{k}\left(F_{k}-\phi_{k} F_{k}^{\prime}\right)\right)(\bar{x}, \bar{y}) & =\sum_{k=1}^{p} \partial^{*}\left(w_{k}\left(F_{k}-\phi_{k} F_{k}^{\prime}\right)\right)(\bar{x}, \bar{y}) \\
& =\sum_{k=1}^{p} w_{k}\left(\partial^{*} F_{k}(\bar{x}, \bar{y})-\phi_{k}(\bar{x}, \bar{y}) \partial^{*} F_{k}^{\prime}(\bar{x}, \bar{y})\right)
\end{aligned}
$$

is a convexifactor of $\sum_{k=1}^{p} w_{k}\left(F_{k}-\phi_{k} F_{k}^{\prime}\right)$ at $(\bar{x}, \bar{y})$.

Remark 7. If $f$ admits a convexifactor $\partial^{*} f(\bar{x}, \bar{y})$ which is upper regular, then $V$ will also have an upper regular convexifactor $\partial^{*} V(\bar{x})$, and using Remark $2,-\partial^{*} V(\bar{x})$ will be a lower regular convexifactor of $-V$ at $\bar{x}$. Then, using Remark 1 and Lemma 2.1(ii), $\partial^{*}(f(\bar{x}, \bar{y})-V(\bar{x}))=$ $\partial^{*} f(\bar{x}, \bar{y})-\partial^{*} V(\bar{x}) \times\{0\}$ is a convexifactor of $f-V$ at $(\bar{x}, \bar{y})$.

Now we give an example to illustrate above theorem.

Example 3. Consider the problem

$$
\begin{aligned}
& \underset{x, y}{\operatorname{Minimize}}\left[\frac{F_{1}(x, y)}{F_{1}^{\prime}(x, y)}, \frac{F_{2}(x, y)}{F_{2}^{\prime}(x, y)}\right] \\
& \text { subject to } G(x, y) \leq 0, y \in \psi(x),
\end{aligned}
$$

where for each $x \in \mathbb{R}, \psi(x)$ is the set of optimal solutions to the following optimization problem

$$
\begin{aligned}
& \underset{y}{\operatorname{Minimize}} f(x, y) \\
& \text { subject to } g(x, y) \leq 0,
\end{aligned}
$$

where $F_{1}, F_{2}, F_{1}^{\prime}, F_{2}^{\prime}, G, f, g: \mathbb{R} \times \mathbb{R} \rightarrow \mathbb{R}$ are defined by

$$
F_{1}(x, y):= \begin{cases}x+y, & x \geq 0, y \geq 0 \\ x+y^{2}, & x \geq 0, y<0 \\ |x|+y^{2}, & x<0, y \leq 0 \\ y, & x<0, y>0\end{cases}
$$




$$
\begin{aligned}
& F_{2}(x, y):= \begin{cases}x+|y|, & x \geq 0, y \in \mathbb{R}, \\
x^{2}+|y|, & x<0, y \in \mathbb{R},\end{cases} \\
& F_{1}^{\prime}(x, y):= \begin{cases}1+x^{2}, & x \in \mathbb{R}, y \geq 0 \sim\{x<0, y>0 \& x=0, y>0\} \\
1+2 y+3 y^{2}, & x \in \mathbb{R}, y<0, \\
1+2 y^{2}, & x \leq 0, y>0,\end{cases} \\
& F_{2}^{\prime}(x, y):= \begin{cases}1+x+y^{2}, & x \geq 0, y \geq 0 ; x>0, y<0, \\
1+2 x+3 x^{2}, & x<0, y \in \mathbb{R} ; x=0, y<0\end{cases} \\
& G(x, y):=y, \\
& f(x, y):= \begin{cases}e^{x}+y+y^{2}, & x \in \mathbb{R}, y \leq 0 \\
x+y, & x \in \mathbb{R}, y>0\end{cases} \\
& g(x, y):=y^{2}+y
\end{aligned}
$$

The corresponding value function for lower-level problem is given by $V(x):=e^{x}$, for all $x \in \mathbb{R}$ and the set $\psi(x)$ of optimal solutions to the lower-level problem is given by

$$
\psi(x):=\{0\}, \quad \text { for all } x \in \mathbb{R} .
$$

There exists no feasible solution $(x, y) \in E$ such that the following hold

$$
\frac{F_{1}(x, y)}{F_{1}^{\prime}(x, y)}<\frac{F_{1}(\bar{x}, \bar{y})}{F_{1}^{\prime}(\bar{x}, \bar{y})} \quad \text { and } \quad \frac{F_{2}(x, y)}{F_{2}^{\prime}(x, y)}<\frac{F_{2}(\bar{x}, \bar{y})}{F_{2}^{\prime}(\bar{x}, \bar{y})}
$$

where $E:=\left\{(x, y): x \in \mathbb{R},-\frac{1}{2} \leq y \leq 0\right\}$.

Thus, $(\bar{x}, \bar{y})=(0,0)$ is a weak efficient solution of the problem.

It can be seen that $F_{1}, F_{2}$ admit CFs $\partial^{*} F_{1}(0,0)=\{(1,1),(0,1),(1,0),(-1,0)\}, \partial^{*} F_{2}(0,0)=$ $\{(1,1),(0,-1),(1,-1),(0,1)\}$ respectively at $(0,0)$ which are upper regular and $F_{1}^{\prime}, F_{2}^{\prime}$ admit CFs $\partial^{*} F_{1}^{\prime}(0,0)=\{(0,0),(0,1),(0,2)\}, \partial^{*} F_{2}^{\prime}(0,0)=\{(1,0),(2,0)\}$ respectively at $(0,0)$ which are lower regular. $G$ and $g$ admit CFs $\partial^{*} G(0,0)=\{(0,1)\}, \partial^{*} g(0,0)=\{(0,1)\}$ respectively at $(0,0)$. $f$ admits a $\mathrm{CF} \partial^{*} f(0,0)=\{(1,1)\}$ at $(0,0)$ which is upper regular. $\partial^{*} V(0) \times\{0\}=\{(1,0)\}$, $\operatorname{conv}\left(\partial^{*} f(0,0)-\partial^{*} V(0) \times\{0\}\right)=\{(0,1)\}$.

$$
\phi_{1}(0,0)=0, \quad \phi_{2}(0,0)=0 .
$$

$\partial^{*}$-MOFBLPP CQ is satisfied at $(\bar{x}, \bar{y})=(0,0)$.

Then, there exist scalars $w_{1}^{\prime}=\frac{1}{3}, w_{2}^{\prime}=\frac{1}{3}, \gamma=\frac{1}{3}, \mu=0, \tau=0$ with $w_{1}^{\prime}+w_{2}^{\prime}+\gamma+\mu+\tau=1$ such that

$$
(0,0) \in \operatorname{cl}\left[\begin{array}{c}
\sum_{k=1}^{2} w_{k}^{\prime}\left\{\operatorname{conv} \partial^{*} F_{k}(\bar{x}, \bar{y})-\phi_{k}(\bar{x}, \bar{y}) \operatorname{conv} \partial^{*} F_{k}^{\prime}(\bar{x}, \bar{y})\right\} \\
+\gamma\left(\operatorname{conv}\left(\partial^{*} f(\bar{x}, \bar{y})-\partial^{*} V(\bar{x}) \times\{0\}\right)\right) \\
+\tau \operatorname{conv} \partial^{*} G(\bar{x}, \bar{y})+\mu \operatorname{conv} \partial^{*} g(\bar{x}, \bar{y})
\end{array}\right] .
$$

\section{Sufficient optimality conditions}

Now we prove sufficient optimality conditions for MOFBLPP. We shall now onwards assume that MOFBLPP has at least one optimal solution. 
Theorem 6.1. Let $(\bar{x}, \bar{y}) \in E$. Assume that $\partial^{*} F_{k}(\bar{x}, \bar{y})$ are convexifactors of $F_{k}, k=1,2, \ldots, p$ at $(\bar{x}, \bar{y})$ which are upper regular and $\partial^{*} F_{k}^{\prime}(\bar{x}, \bar{y})$ are convexifactors of $F_{k}^{\prime}, k=1,2, \ldots, p$ at $(\bar{x}, \bar{y})$ which are upper regular if $\phi_{k}(\bar{x}, \bar{y})>0$ and lower regular if $\phi_{k}(\bar{x}, \bar{y})<0, k=1,2, \ldots, p$. Suppose that $\partial^{*} \varphi_{k}(x, y)=\partial^{*}\left(F_{k}-\phi_{k} F_{k}^{\prime}\right)(x, y)=\partial^{*} F_{k}(x, y)-\phi_{k}(\bar{x}, \bar{y}) \partial^{*} F_{k}^{\prime}(x, y), k=1,2, \ldots, p$. Let $\varphi_{k}, k=1,2, \ldots, p$ be $\partial^{*}$-asymptotic pseudoconvex, $f-V, g_{i}, i \in I(\bar{x}, \bar{y})$ and $G_{j}, j \in J(\bar{x}, \bar{y})$ be $\partial^{*}$-asymptotic quasiconvex at $(\bar{x}, \bar{y})$. Suppose that there exist scalars $w_{k}^{\prime} \geq 0$ (not all zero), $k=1,2, \ldots, p, \gamma \geq 0, \tau_{j} \geq 0, j \in J, \mu_{i} \geq 0, i \in I$ with $\sum_{k=1}^{p} w_{k}^{\prime}+\gamma+\sum_{j \in J} \tau_{j}+\sum_{i \in I} \mu_{i}=1$ such that the following condition is satisfied

$$
(0,0) \in \operatorname{cl}\left[\begin{array}{c}
\sum_{k=1}^{p} w_{k}^{\prime}\left\{\operatorname{conv} \partial^{*} F_{k}(\bar{x}, \bar{y})-\phi_{k}(\bar{x}, \bar{y}) \operatorname{conv} \partial^{*} F_{k}^{\prime}(\bar{x}, \bar{y})\right\} \\
+\gamma\left(\operatorname{conv}\left(\partial^{*} f(\bar{x}, \bar{y})-\partial^{*} V(\bar{x}) \times\{0\}\right)\right) \\
+\sum_{j \in J} \tau_{j} \operatorname{conv} \partial^{*} G_{j}(\bar{x}, \bar{y})+\sum_{i \in I} \mu_{i} \operatorname{conv} \partial^{*} g_{i}(\bar{x}, \bar{y})
\end{array}\right] .
$$

Then, $(\bar{x}, \bar{y})$ is a weak efficient solution of MOFBLPP.

Proof. Suppose that $(\bar{x}, \bar{y})$ is not a weak efficient solution of MOFBLPP, then by Remark $3,(\bar{x}, \bar{y})$ is not a weak efficient solution of RMOFBLPP.

Thus, there exists a feasible solution $(x, y) \in E$ such that

$$
\frac{F_{k}(x, y)}{F_{k}^{\prime}(x, y)}<\frac{F_{k}(\bar{x}, \bar{y})}{F_{k}^{\prime}(\bar{x}, \bar{y})}, \quad k=1,2, \ldots, p
$$

which implies that $F_{k}(x, y)-\phi_{k}(\bar{x}, \bar{y}) F_{k}^{\prime}(x, y)<0, k=1,2, \ldots, p$.

Since $F_{k}(\bar{x}, \bar{y})-\phi_{k}(\bar{x}, \bar{y}) F_{k}^{\prime}(\bar{x}, \bar{y})=0, k=1,2, \ldots, p$, we have

$$
F_{k}(x, y)-\phi_{k}(\bar{x}, \bar{y}) F_{k}^{\prime}(x, y)<F_{k}(\bar{x}, \bar{y})-\phi_{k}(\bar{x}, \bar{y}) F_{k}^{\prime}(\bar{x}, \bar{y}), \quad k=1,2, \ldots, p .
$$

That is, we have

$$
\varphi_{k}(x, y)<\varphi_{k}(\bar{x}, \bar{y}), \quad k=1,2, \ldots, p .
$$

Using $\partial^{*}$-asymptotic pseudoconvexity of $\varphi_{k}, k=1,2, \ldots, p$, we get

$$
\left\langle\xi_{k}^{0},(x-\bar{x}, y-\bar{y})\right\rangle<0 \quad \text { for all } \xi_{k}^{0} \in \operatorname{clconv} \partial^{*} \varphi_{k}(\bar{x}, \bar{y}), k=1,2, \ldots, p .
$$

Now,

$$
\begin{aligned}
\operatorname{conv} \partial^{*} \varphi_{k}(\bar{x}, \bar{y}) & =\operatorname{conv} \partial^{*}\left(F_{k}-\phi_{k} F_{k}^{\prime}\right)(\bar{x}, \bar{y}) \\
& =\operatorname{conv}\left\{\partial^{*} F_{k}(\bar{x}, \bar{y})-\phi_{k}(\bar{x}, \bar{y}) \partial^{*} F_{k}^{\prime}(\bar{x}, \bar{y})\right\}, \quad k=1,2, \ldots, p
\end{aligned}
$$

Using the convex hull property of subsets $A_{1}$ and $A_{2}$ of $\mathbb{R}^{n_{1}}, \operatorname{conv}\left(A_{1}+A_{2}\right)=\operatorname{conv}\left(A_{1}\right)+\operatorname{conv}\left(A_{2}\right)$ and of a subset $A$ of $\mathbb{R}^{n_{1}}, \operatorname{conv}(\lambda A)=\lambda \operatorname{conv}(A), \lambda \in \mathbb{R}$, we get

$$
\begin{aligned}
& \operatorname{conv}\left\{\partial^{*} F_{k}(\bar{x}, \bar{y})-\phi_{k}(\bar{x}, \bar{y}) \partial^{*} F_{k}^{\prime}(\bar{x}, \bar{y})\right\} \\
& \quad=\operatorname{conv} \partial^{*} F_{k}(\bar{x}, \bar{y})-\phi_{k}(\bar{x}, \bar{y}) \operatorname{conv} \partial^{*} F_{k}^{\prime}(\bar{x}, \bar{y}), \quad k=1,2, \ldots, p .
\end{aligned}
$$

Thus, combining (6.3) and (6.4), we get

$$
\operatorname{conv} \partial^{*} \varphi_{k}(\bar{x}, \bar{y})=\operatorname{conv} \partial^{*} F_{k}(\bar{x}, \bar{y})-\phi_{k}(\bar{x}, \bar{y}) \operatorname{conv} \partial^{*} F_{k}^{\prime}(\bar{x}, \bar{y}), \quad k=1,2, \ldots, p .
$$


Now, by (6.1), there exist

$$
\begin{aligned}
& \xi_{k}^{n} \in \operatorname{conv} \partial^{*} F_{k}(\bar{x}, \bar{y}), \xi_{k}^{\prime n} \in \operatorname{conv} \partial^{*} F_{k}^{\prime}(\bar{x}, \bar{y}), \quad k=1,2, \ldots, p, \\
& \zeta^{n} \in \operatorname{conv}\left(\partial^{*} f(\bar{x}, \bar{y})-\partial^{*} V(\bar{x}) \times\{0\}\right), \\
& \eta_{i}^{n} \in \operatorname{conv} \partial^{*} g_{i}(\bar{x}, \bar{y}), \quad i \in I, \quad \gamma_{j}^{n} \in \operatorname{conv} \partial^{*} G_{j}(\bar{x}, \bar{y}), \quad j \in J \quad \text { such that } \\
& \lim _{n \rightarrow \infty}\left[\sum_{k=1}^{p} w_{k}^{\prime}\left(\xi_{k}^{n}-\phi_{k}(\bar{x}, \bar{y}) \xi_{k}^{\prime n}\right)+\gamma \zeta^{n}+\sum_{i \in I} \mu_{i} \eta_{i}^{n}+\sum_{j \in J} \tau_{j} \gamma_{j}^{n}\right]=(0,0) .
\end{aligned}
$$

Then, for all $(x, y) \in E$, we have

$$
\lim _{n \rightarrow \infty}\left[\begin{array}{l}
\sum_{k=1}^{p} w_{k}^{\prime}\left\langle\left(\xi_{k}^{n}-\phi_{k}(\bar{x}, \bar{y}) \xi_{k}^{\prime} n\right),(x-\bar{x}, y-\bar{y})\right\rangle+\gamma\left\langle\zeta^{n},(x-\bar{x}, y-\bar{y})\right\rangle \\
+\sum_{i \in I} \mu_{i}\left\langle\eta_{i}^{n},(x-\bar{x}, y-\bar{y})\right\rangle+\sum_{j \in J} \tau_{j}\left\langle\gamma_{j}^{n},(x-\bar{x}, y-\bar{y})\right\rangle
\end{array}\right]=0 .
$$

Now, from $(6.2)$, since $\xi_{k}^{0} \in \operatorname{clconv} \partial^{*} \varphi_{k}(\bar{x}, \bar{y}), k=1,2, \ldots, p$, there exists $\xi_{k}^{0 n} \in \operatorname{conv} \partial^{*} \varphi_{k}(\bar{x}, \bar{y})$, $k=1,2, \ldots, p$ such that

$$
\lim _{n \rightarrow \infty} \xi_{k}^{0 n}=\xi_{k}^{0}, \quad k=1,2, \ldots, p
$$

Using above in (6.2), we get

$$
\lim _{n \rightarrow \infty}\left\langle\xi_{k}^{0 n},(x-\bar{x}, y-\bar{y})\right\rangle<0, \quad \text { for some } \xi_{k}^{0 n} \in \operatorname{conv} \partial^{*} \varphi_{k}(\bar{x}, \bar{y}), \quad k=1,2, \ldots, p .
$$

Thus, for every $\xi_{k}^{0} \in \operatorname{clconv} \partial^{*} \varphi_{k}(\bar{x}, \bar{y}), k=1,2, \ldots, p$, there exists $\xi_{k}^{0 n} \in \operatorname{conv} \partial^{*} \varphi_{k}(\bar{x}, \bar{y}), k=$ $1,2, \ldots, p$ such that $(6.7)$ holds.

In particular, it is true for $\xi_{k}^{0} \in \operatorname{clconv} \partial^{*} \varphi_{k}(\bar{x}, \bar{y}), k=1,2, \ldots, p$ for which there exists $\xi_{k}^{0 n} \in \operatorname{conv} \partial^{*} \varphi_{k}(\bar{x}, \bar{y})$ such that

$$
\xi_{k}^{0}=\lim _{n \rightarrow \infty} \xi_{k}^{0 n}=\lim _{n \rightarrow \infty}\left(\xi_{k}^{n}-\phi_{k}(\bar{x}, \bar{y}) \xi_{k}^{\prime n}\right),
$$

where $\xi_{k}^{n} \in \operatorname{conv} \partial^{*} F_{k}(\bar{x}, \bar{y}), \xi_{k}^{\prime n} \in \operatorname{conv} \partial^{*} F_{k}^{\prime}(\bar{x}, \bar{y}), k=1,2, \ldots, p$.

Thus, we get

$$
\begin{aligned}
& \lim _{n \rightarrow \infty}\left[\sum_{k=1}^{p} w_{k}^{\prime}\left\langle\left(\xi_{k}^{n}-\phi_{k}(\bar{x}, \bar{y}) \xi_{k}^{\prime n}\right),(x-\bar{x}, y-\bar{y})\right\rangle\right]<0 \text { as } \xi_{k}^{n} \in \operatorname{conv} \partial^{*} F_{k}(\bar{x}, \bar{y}), \\
& \xi_{k}^{\prime n} \in \operatorname{conv} \partial^{*} F_{k}^{\prime}(\bar{x}, \bar{y}), w_{k}^{\prime} \geq 0 \text { (not all zero). }
\end{aligned}
$$

Since $(x, y)$ is feasible for RMOFBLPP, we have

$$
f(x, y)-V(x) \leq 0 \leq f(\bar{x}, \bar{y})-V(\bar{x}) .
$$

By $\partial^{*}$-asymptotic quasiconvexity of $f-V$, we get

$$
\langle\zeta,(x-\bar{x}, y-\bar{y})\rangle \leq 0, \quad \text { for all } \zeta \in \operatorname{cl}\left(\operatorname{conv}\left(\partial^{*} f(\bar{x}, \bar{y})-\partial^{*} V(\bar{x}) \times\{0\}\right)\right) .
$$

In particular, it is true for $\zeta$ such that $\zeta=\lim _{n \rightarrow \infty} \zeta^{n}$, where $\zeta^{n} \in \operatorname{conv}\left(\partial^{*} f(\bar{x}, \bar{y})-\partial^{*} V(\bar{x}) \times\{0\}\right)$. 
That is,

$$
\lim _{n \rightarrow \infty}\left\langle\zeta^{n},(x-\bar{x}, y-\bar{y})\right\rangle \leq 0, \text { as } \zeta^{n} \in \operatorname{conv}\left(\partial^{*} f(\bar{x}, \bar{y})-\partial^{*} V(\bar{x}) \times\{0\}\right),
$$

which gives

$$
\lim _{n \rightarrow \infty} \gamma\left\langle\zeta^{n},(x-\bar{x}, y-\bar{y})\right\rangle \leq 0, \text { as } \zeta^{n} \in \operatorname{conv}\left(\partial^{*} f(\bar{x}, \bar{y})-\partial^{*} V(\bar{x}) \times\{0\}\right) \text { and } \gamma \geq 0 \text {. }
$$

Now, for each $i \in I(\bar{x}, \bar{y}), g_{i}(x, y) \leq 0=g_{i}(\bar{x}, \bar{y})$.

Using $\partial^{*}$-asymptotic quasiconvexity of $g_{i}, i \in I(\bar{x}, \bar{y})$, we have

$$
\left\langle\eta_{i},(x-\bar{x}, y-\bar{y})\right\rangle \leq 0, \quad \text { for all } \eta_{i} \in \operatorname{clconv} \partial^{*} g_{i}(\bar{x}, \bar{y}), i \in I(\bar{x}, \bar{y}) .
$$

Then, as earlier, we have

$$
\lim _{n \rightarrow \infty}\left\langle\eta_{i}^{n},(x-\bar{x}, y-\bar{y})\right\rangle \leq 0, \text { as } \eta_{i}^{n} \in \operatorname{conv} \partial^{*} g_{i}(\bar{x}, \bar{y}), \quad \text { for } i \in I(\bar{x}, \bar{y}) .
$$

Thus, we have

$$
\lim _{n \rightarrow \infty}\left\langle\sum_{i \in I(\bar{x}, \bar{y})} \mu_{i} \eta_{i}^{n},(x-\bar{x}, y-\bar{y})\right\rangle \leq 0, \text { as } \mu_{i} \geq 0 \text { and } \eta_{i}^{n} \in \operatorname{conv} \partial^{*} g_{i}(\bar{x}, \bar{y}) .
$$

Taking $\mu_{i}=0, i \notin I(\bar{x}, \bar{y})$, we get

$$
\lim _{n \rightarrow \infty}\left\langle\sum_{i \in I} \mu_{i} \eta_{i}^{n},(x-\bar{x}, y-\bar{y})\right\rangle \leq 0, \text { as } \mu_{i} \geq 0 \text { and } \eta_{i}^{n} \in \operatorname{conv} \partial^{*} g_{i}(\bar{x}, \bar{y}) .
$$

Similarly, we have

$$
G_{j}(x, y) \leq 0=G_{j}(\bar{x}, \bar{y}), \text { for each } j \in J(\bar{x}, \bar{y}) .
$$

Using $\partial^{*}$-asymptotic quasiconvexity of $G_{j}, j \in J(\bar{x}, \bar{y})$, taking $\tau_{j}=0, j \notin J(\bar{x}, \bar{y})$ and proceeding as above, we get

$$
\lim _{n \rightarrow \infty}\left\langle\sum_{j \in J} \tau_{j} \gamma_{j}^{n},(x-\bar{x}, y-\bar{y})\right\rangle \leq 0 \text { as } \tau_{j} \geq 0, j \in J(\bar{x}, \bar{y}) \text { and } \gamma_{j}^{n} \in \operatorname{conv} \partial^{*} G_{j}(\bar{x}, \bar{y}) .
$$

Adding (6.8), (6.9), (6.10) and (6.11), we get

$$
\lim _{n \rightarrow \infty}\left[\begin{array}{c}
\sum_{k=1}^{p} w_{k}^{\prime}\left\langle\left(\xi_{k}^{n}-\phi_{k}(\bar{x}, \bar{y}) \xi_{k}^{\prime n}\right),(x-\bar{x}, y-\bar{y})\right\rangle+\gamma\left\langle\zeta^{n},(x-\bar{x}, y-\bar{y})\right\rangle \\
+\sum_{i \in I} \mu_{i}\left\langle\eta_{i}^{n},(x-\bar{x}, y-\bar{y})\right\rangle+\sum_{j \in J} \tau_{j}\left\langle\gamma_{j}^{n},(x-\bar{x}, y-\bar{y})\right\rangle
\end{array}\right]<0,
$$

which is contradiction to $(6.6)$. Hence $(\bar{x}, \bar{y})$ is a weak efficient solution of MOFBLPP.

Following example illustrates the above theorem.

Example 4. Consider the problem

$$
\underset{x, y}{\operatorname{Minimize}}\left[\frac{F_{1}(x, y)}{F_{1}^{\prime}(x, y)}, \frac{F_{2}(x, y)}{F_{2}^{\prime}(x, y)}\right]
$$




$$
\text { subject to } G(x, y) \leq 0, y \in \psi(x),
$$

where for each $x \in \mathbb{R}, \psi(x)$ is the set of optimal solutions to the following optimization problem

$$
\begin{aligned}
& \underset{y}{\operatorname{Minimize}} f(x, y) \\
& \text { subject to } g(x, y) \leq 0,
\end{aligned}
$$

where $F_{1}, F_{2}, F_{1}^{\prime}, F_{2}^{\prime}, G, f, g: \mathbb{R} \times \mathbb{R} \rightarrow \mathbb{R}$ are defined by

$$
\begin{aligned}
& F_{1}(x, y):= \begin{cases}\frac{-|y|}{2}, & x \geq 0, y \geq 0 \\
\frac{-x-y}{2}, & x<0, y \in \mathbb{R} ; x=0, y<0, \\
\frac{|y|}{2}, & x>0, y<0\end{cases} \\
& F_{2}(x, y):= \begin{cases}\frac{-|y|}{2}, & x>0, y>0 ; x=0, y \geq 0 \\
\frac{|y|}{2}, & x \geq 0, y<0 \\
\frac{x^{2}+y^{2}}{2}, & x>0, y=0 \\
\frac{-x-y}{2}, & x<0, y \in \mathbb{R}\end{cases} \\
& F_{1}^{\prime}(x, y):= \begin{cases}1-|x|-y, & x \geq 0, y \geq 0 \\
1+|x|-y, & x<0, y \geq 0 \\
1+|x|, & x \leq 0, y<0 \\
1-|x|, & x>0, y<0\end{cases} \\
& F_{2}^{\prime}(x, y):= \begin{cases}1-x-y, & x \geq 0, y \geq 0 \\
1-x-y, & x<0, y>0 \\
1-x, & x<0, y \leq 0 \\
1-x, & x \geq 0, y<0\end{cases} \\
& G(x, y):=x, \\
& f(x, y):= \begin{cases}x+y^{2}, & x \in \mathbb{R}, y \leq 0 \sim\{x<0, y=0\} \\
x+y, & x \geq 0, y>0 ; x<0, y \geq 0\end{cases} \\
& g(x, y):=y^{2}+y \text {. }
\end{aligned}
$$

The corresponding value function for lower-level problem is given by

$$
V(x):=x \text { for all } x \in \mathbb{R}
$$

and the set $\psi(x)$ of optimal solutions to the lower-level problem is given by

$$
\psi(x):=\{0\}, \quad \text { for all } x \in \mathbb{R} .
$$

There exists no feasible solution $(x, y) \in E$ such that the following hold

$$
\frac{F_{1}(x, y)}{F_{1}^{\prime}(x, y)}<\frac{F_{1}(\bar{x}, \bar{y})}{F_{1}^{\prime}(\bar{x}, \bar{y})} \quad \text { and } \quad \frac{F_{2}(x, y)}{F_{2}^{\prime}(x, y)}<\frac{F_{2}(\bar{x}, \bar{y})}{F_{2}^{\prime}(\bar{x}, \bar{y})}
$$

where $E:=\{(x, 0): x \leq 0\}$. Thus, $(\bar{x}, \bar{y})=(0,0)$ is a weak efficient solution of the problem.

It can be seen that $\varphi_{1}, \varphi_{2}$ are $\partial^{*}$-asymptotic pseudoconvex at $(0,0)$ with respect to

$$
\partial^{*} \varphi_{1}(0,0)=\partial^{*} F_{1}(0,0)
$$




$$
\begin{aligned}
& =\left\{\left(\frac{-1}{2}+\frac{1}{n}, \frac{-1}{2}+\frac{1}{n}\right),\left(0+\frac{1}{n}, \frac{-1}{2}+\frac{1}{n}\right)\right\} \cup\left\{\left(-\frac{1}{2},-\frac{1}{2}\right),\left(0,-\frac{1}{2}\right)\right\}, \\
\partial^{*} \varphi_{2}(0,0) & =\partial^{*} F_{2}(0,0) \\
& =\left\{\left(0+\frac{1}{n}, \frac{-1}{2}+\frac{1}{n}\right),\left(\frac{-1}{2}+\frac{1}{n}, \frac{-1}{2}+\frac{1}{n}\right)\right\} \cup\left\{\left(0, \frac{-1}{2}\right),\left(\frac{-1}{2}, \frac{-1}{2}\right)\right\}
\end{aligned}
$$

respectively. $\phi_{1}(0,0)=0, \phi_{2}(0,0)=0 . G$ and $g$ are $\partial^{*}$-asymptotic quasiconvex at $(0,0)$ with respect to $\partial^{*} G(0,0)=\left\{\left(1+\frac{1}{n}, 0\right)\right\} \cup\{(1,0)\}$ and $\partial^{*} g(0,0)=\left\{\left(0,1+\frac{1}{n}\right)\right\} \cup\{(0,1)\}$ respectively. $f-V$ is $\partial^{*}$-asymptotic quasiconvex at $(0,0)$ with respect to $\partial^{*} f(0,0)-\partial^{*} V(0) \times\{0\}=$ $\left\{\left(0+\frac{1}{n}, 1+\frac{1}{n}\right),\left(0+\frac{1}{n}, 0+\frac{1}{n}\right)\right\} \cup\{(0,1),(0,0)\}$.

Then, there exist scalars $w_{1}^{\prime}=\frac{1}{4}, w_{2}^{\prime}=\frac{1}{4}, \gamma=\frac{1}{4}, \mu=\frac{1}{4}, \tau=0$ with $w_{1}^{\prime}+w_{2}^{\prime}+\gamma+\mu+\tau=1$ such that

$$
(0,0) \in \operatorname{cl}\left[\begin{array}{c}
\sum_{k=1}^{2} w_{k}^{\prime}\left\{\operatorname{conv} \partial^{*} F_{k}(\bar{x}, \bar{y})-\phi_{k}(\bar{x}, \bar{y}) \operatorname{conv} \partial^{*} F_{k}^{\prime}(\bar{x}, \bar{y})\right\} \\
+\gamma\left(\operatorname{conv}\left(\partial^{*} f(\bar{x}, \bar{y})-\partial^{*} V(\bar{x}) \times\{0\}\right)\right) \\
+\tau \operatorname{conv} \partial^{*} G(\bar{x}, \bar{y})+\mu \operatorname{conv} \partial^{*} g(\bar{x}, \bar{y})
\end{array}\right] .
$$

\section{Conclusions}

In this article, necessary and sufficient optimality conditions using the concept of convexifactors are developed for multiobjective fractional bilevel programming problem. For that, a suitable $\mathrm{CQ}$ in terms of convexifactors has been introduced.Under the assumptions of asymptotic pseudoconvexity and asymptotic quasiconvexity on the functions, sufficient optimality conditions are established for the problem.

Acknowledegments Author would like to thank the anonymous referee for valuable comments and suggestions which has improved the paper.

\section{References}

[1] J. F. Bard, Optimality conditions for the bilevel programming problem, Naval Research Logistics Quarterly 31 (1984), 13-26.

[2] J. F. Bard, Some properties of the bilevel programming problem, Journal of Optimization Theory and Applications 68 (1991), 371-378.

[3] J. F. Bard, Practical Bilevel Optimization: Algorithms and Applications, Kluwer Academic Publications, The Netherlands (1998).

[4] C. R. Bector, S. Chandra, J. Dutta, Principles of Optimization Theory, Narosa Publications (2005).

[5] H. Bonnel, Optimality conditions for the semivectorial bilevel optimization problem, Pacific Journal of Optimization 2(3) (2006), 447-467.

[6] H. Bonnel, J. Morgan, Semivectorial bilevel optimization problem; penalty approach, Journal of Optimization Theory and Applications 131(3) (2006), 365-382. 
[7] H. Bonnel, J. Morgan, Optimality conditions for semivectorial bilevel convex optimal control problem, In: H. Bauschke, M. Thera (eds.), Computational and Analytical Mathematics, Springer Proceeding in Mathematics 50 (2013), 45-78.

[8] K. Bouibed, S. Hachem, M. S. Radjef, Global efficiency for multiobjective bilevel programming problems under generalized invexity, Journal of Applied Mathematics and Computing, 53 , no. 1-2, 507-530 (2017)

[9] H. I. Calvete, C. Gale, The bilevel linear/ linear fractional programming problem, European Journal of Operational Research 114 (1) (1999), 188-197.

[10] H. I. Calvete, C.. Gale, A note on 'Bi-Level linear fractional programming problem', European Journal of Operational Research 152(1) (2004(a)), 296-299.

[11] H. I. Calvete, C. Gale, Optimality conditions for the linear fractional/ quadratic bilevel problem, Monografias del Seminario Matematico Garcia de Galdeano 31 (2004), 285-294.

[12] S. Dempe, A necessary and sufficient optimality condition for bilevel programming problem, Optimization 25 (1992), 341-354.

[13] S. Dempe, First order necessary optimality conditions for general bilevel programming problems, Journal of Optimization Theory and Applications 95 (1997), 735-739.

[14] S. Dempe, Foundations of Bilevel Programming, Kluwer Academic Publishers (2002).

[15] S. Dempe, J. Dutta, B. S. Mordukhovich, New necessary optimality conditions in optimistic bilevel programming, Optimization 56 (2007), 577-604.

[16] V. F. Demyanov, Convexification and concavification of positively homogeneous function by the same family of linear functions, Report 3.208,802, Universita di pisa (1994).

[17] J. Dutta, S. Chandra, Convexifactors, generalized convexity and optimality conditions, Journal of Optimization Theory and Applications 113 (2002), 41-65.

[18] J. Dutta, S. Chandra, Convexifactors, generalized convexity and vector optimization, Optimization 53 (2004), 77-94.

[19] N. Gadhi, S. Dempe, Necessary optimality conditions and a new approach to multiobjective bilevel optimization problems, Journal of Optimization Theory and Applications 155(1) (2014), 100-114.

[20] V. Jeyakumar, D. T. Luc, Nonsmooth calculus, maximality and monotonicity of convexificators, Journal of Optimization Theory and Applications 101 (1999), 599-621.

[21] A. Jourani, Constraint qualifications and lagrange multipliers in nondifferentiable programming problems, Journal of Optimization Theory and Applications 81(3) (1994), 533-548.

[22] A. Kabgani, M. Soleimani-damaneh, Characterizations of (weakly/properly/roboust) efficient solutions in nonsmooth semi-infinite multiobjective optimization using convexificators, Optimization 67 (2) (2018), 217-235.

[23] A. Kabgani, M. Soleimani-damaneh, The Relationships between convexificators and Greensberg-Pierskalla subdifferentials for quasiconvex functions, Numerical Functional Analysis and Optimization 38 (12) (2017), 1548-1563. 
[24] A. Kabgani, M. Soleimani-damaneh, M. Zamani, Optimality conditions in optimization problems with convex feasible set using convexifactors, Mathematical Methods of Operations Research 86 (2017), 103-121.

[25] B. Kohli, Optimality conditions for optimistic bilevel programming problem using convexifactors, Journal of Optimization Theory and Applications 152 (3) (2012), 632-651.

[26] B. Kohli, Necessary and sufficient optimality conditions using convexifactors for mathematical programs with equilibrium constraints, Rairo Operations Research 53 (2019), 1617-1632.

[27] X. F. Li, J. Z. Zhang, Necessary optimality conditions in terms of convexificators in lipschitz optimization, Journal of Optimization Theory and Applications 131 (2006), 429-452.

[28] D. V. Luu, Optimality condition for local efficient solutions of vector equilibrium problems via convexificators and applications, Journal of Optimization Theory and Applications $\mathbf{1 7 1}$ (2) (2016), 643-665.

[29] D.V. Luu, T.T. Mai, Efficiency conditions for multiobjective bilevel programming problems via convexificators, J. Nonlinear Var. Anal. 4(3) (2020), 399-414.

[30] D. V. Luu, P. T. Linh, Optimality and duality for nonsmooth multiobjective fractional problems using convexificators, J. Nonlinear Funct. Anal. (2021), Article ID 1.

[31] J. V. Outrata, On necessary optimality conditions for stackelberg problems, Journal of Optimization Theory and Applications 76 (1993), 306-320.

[32] S. K. Suneja, B. Kohli, Optimality and duality results for bilevel programming problem using convexifactors, Journal of Optimization Theory and Applications 150 (2011), 1-19.

[33] S. K. Suneja, B. Kohli, Generalized nonsmooth cone convexity in terms of convexifactors in vector optimization, Opsearch 50 (1) (2013), 89-105.

[34] S. K. Suneja, B. Kohli, Duality for multiobjective fractional programming problem using convexifactors, Mathematical Sciences 7:6 (2013), 8 pages.

[35] L. T. Tung, Karush-Kuhn-Tucker optimality conditions for nonsmooth multiobjective semidefinite and semi-infinite programming, J. Appl. Numer. Optim. 1(1) (2019), 63-75.

[36] J. J. Ye, Necessary optimality conditions for multiobjective bilevel programs, Mathematics of Operations Research 36 (1) (2011), 165-184.

[37] J. J. Ye, Nondifferentiable multiplier rules for optimization and bilevel optimization problems, Siam Journal of Optimization 15 (2004), 252-274.

[38] J. J. Ye, Constraint qualifications and KKT conditions for bilevel programming problems, Mathematics of Operations Research 31 (2006), 811-824.

Bhawna Kohli Department of Mathematics, P.G.D.A.V. College, University of Delhi, Delhi110065, India

E-mail: bhawna_kohli@rediffmail.com 